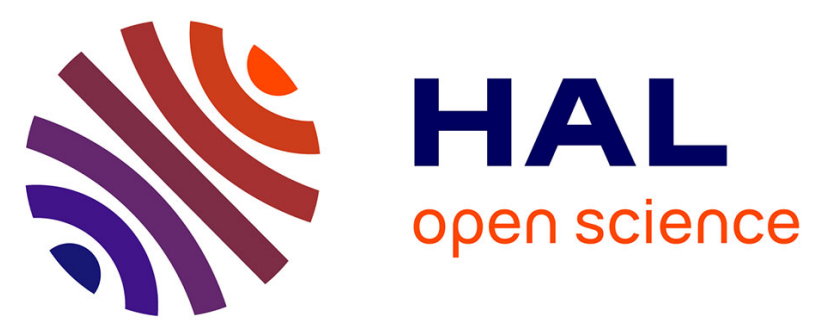

\title{
RECHERCHE DE PRISE DE MASSE MUSCULAIRE ET DYSMORPHIE MUSCULAIRE CHEZ LES BODYBUILDERS : UNE REVUE DE LA LITTÉRATURE ANGLOPHONE
}

Lisa Chaba, Stéphanie Scoffier-Mériaux, Vanessa Lentillon-Kaestner, Fabienne d'Arripe-Longueville

\section{To cite this version:}

Lisa Chaba, Stéphanie Scoffier-Mériaux, Vanessa Lentillon-Kaestner, Fabienne d'Arripe-Longueville. RECHERCHE DE PRISE DE MASSE MUSCULAIRE ET DYSMORPHIE MUSCULAIRE CHEZ LES BODYBUILDERS : UNE REVUE DE LA LITTÉRATURE ANGLOPHONE. Deviant Behavior, 2018, 119, pp.15. 10.3917/sta.119.0065 . hal-02524857

\section{HAL Id: hal-02524857 \\ https://hal.univ-cotedazur.fr/hal-02524857}

Submitted on 6 Apr 2020

HAL is a multi-disciplinary open access archive for the deposit and dissemination of scientific research documents, whether they are published or not. The documents may come from teaching and research institutions in France or abroad, or from public or private research centers.
L'archive ouverte pluridisciplinaire HAL, est destinée au dépôt et à la diffusion de documents scientifiques de niveau recherche, publiés ou non, émanant des établissements d'enseignement et de recherche français ou étrangers, des laboratoires publics ou privés. 
Recherche de prise de masse musculaire et dysmorphie musculaire

chez les bodybuilders : une revue

de la littérature anglophone

The drive for muscularity

and muscle dysmorphia in bodybuilders:

English literature review

\author{
LISA CHABA \\ Haute .cole P.dagogique du canton de \\ Vaud, Lausanne, Suisse (HEP-VD) \\ Universit. C.te d'Azur, LAMHESS, France \\ ST.PHANIE SCOFFIER-M.RIAUX \\ Universit. C.te d'Azur, LAMHESS, France \\ VANESSA LENTILLON-KAESTNER \\ Haute .cole P.dagogique du canton de \\ Vaud, Lausanne, Suisse (HEP-VD) \\ FABIENNE D'ARRIPE-LONGUEVILLE \\ Universit. C.te d'Azur, LAMHESS, France \\ La correspondance concernant cet article \\ pourra tre adress.e. Lisa Chaba, \\ Avenue de Cour 25, 1014 Lausanne, \\ Suisse. E-mail : lisa.chaba@ hepl.ch \\ Phone : +33 (0)6 47800784 . \\ Direct : +41 213163287
}


Lisa Chaba

2 Haute Ecole Pédagogique du canton de Vaud, Lausanne, Suisse (HEP-VD)

3 Université Côte d'Azur, LAMHESS, France

4

$5 \quad$ Stéphanie Scoffier-Mériaux

6 Université Côte d'Azur, LAMHESS, France

7

8 Vanessa Lentillon-Kaestner

9 Haute Ecole Pédagogique du canton de Vaud, Lausanne, Suisse (HEP-VD)

10

11 Fabienne d'Arripe-Longueville,

12 Université Côte d'Azur, LAMHESS, France

13

14

15

16

17

18

19 La correspondance concernant cet article pourra être adressée à Lisa Chaba, Avenue de Cour

20 25, 1014 Lausanne, Suisse. E-mail: lisa.chaba@hepl.ch Phone: +33 (0)6 478007 84. Direct:

$21+41213163287$

22

23

24

25 


\section{Introduction}

Depuis les dernières décennies, la population générale est de plus en plus préoccupée par l'apparence physique (e.g., Amadieu, 2002 ; Pope, Olivardia, Gruber, \& Boroweicki, 1999). Les études s'intéressent de plus en plus à l'insatisfaction corporelle chez les hommes due aux répercussions médiatiques (e.g., Baghurst \& Kissinger, 2009 ; Labre, 2005 ; Raevuori et al., 2006). L’idéal masculin véhiculé par les médias se traduit par une silhouette mésomorphique caractérisée par une masse musculaire importante, ayant peu, ou pas de tissus adipeux et des épaules plus larges que le bassin (Labre, 2005). Les hommes aspirent à être forts, athlétiques, et minces, de manière à avoir une définition musculaire apparente (e.g., Labre, 2005). Au cours des 25 dernières années, on observe ainsi une augmentation de la masse musculaire des figurines pour les jeunes garçons et des modèles masculins présentés dans les magazines et dans les films (e.g., Frederick, Fessler, \& Haselton, 2005 ; Pope et al., 1999).

Parallèlement, plusieurs travaux ont montré que les hommes pouvaient être aussi préoccupés par rapport à leur corps, que les femmes (e.g., Frederick et al., 2007 ; Olivardia, Pope, Borowieki, \& Cochrane, 2004 ; Ricciardelli \& McCabe, 2004). Le gouvernement du Québec a réalisé une enquête sociale qui a révélé que $41.7 \%$ des hommes de plus de 15 ans désiraient changer de poids, contre $55.8 \%$ des femmes (Ledoux \& Rivard, 2000). De plus, l'étude de Vartanian, Giant et Passino (2001) a montré que le pourcentage d'hommes qui souhaitaient augmenter leur masse musculaire pouvait aller jusqu'à 85\%.

Les hommes insatisfaits de leur corps vont avoir tendance à s'engager dans des activités sportives permettant de renforcer leur identité, telles que la musculation par exemple (e.g., Gentil, 2015 ; Helms, Aragon, \& Fitschen, 2014). Ainsi, le bodybuilding est un sport où le pratiquant recherche un volume musculaire important, tout en gardant la saillance de ses muscles (Gentil, 2015). Les juges notent les qualités esthétiques obtenues grâce à un 
entraînement en musculation et à un programme nutritif cadré (Mosley, 2009). Cela explique pourquoi les bodybuilders sont des sportifs très touchés par l'insatisfaction corporelle (e.g., Davies, Smith \& Collier, 2011), et potentiellement par le développement des troubles du comportement alimentaire (e.g., Chapman \& Woodman, 2015 ; Mosley, 2009), notamment la dysmorphie musculaire (DM) (e.g., Baghurst \& Kissinger, 2009 ; Cella, Iannaccone, \& Cotrufo, 2012 ; Davies et al., 2011 ; Lantz, Rhea, Cornelius, 2002 ; Leone, Sedory, \& Gray, 2005).

La DM a été définie par Pope, Gruber, Choi, Olivardia, et Phillips (1997) comme étant une préoccupation excessive par rapport à la prise de masse musculaire et à l'adoption de comportements alimentaires et d'exercices physiques associés à la recherche de prise de masse musculaire. La DM a tout d'abord été nommée « anorexie inversée » en raison des points communs avec l'anorexie mentale, aux niveaux cognitif et comportemental (Pope, Katz, \& Hudson, 1993). Ces points communs ont récemment été confirmés dans la revue de Behar et Arancibia (2015). Il a été montré que $29 \%$ des haltérophiles atteints de dysmorphie musculaire ont un passé avec de l'anorexie nerveuse, de la boulimie nerveuse ou des crises de l'alimentation, alors que ce n'est pas le cas chez les haltérophiles sans DM (Olivardia, Pope, \& Hudson, 2000). Les hommes souffrant de DM sont préoccupés par leur image corporelle, c'est-à-dire qu'ils se perçoivent comme frêles et faibles malgré la prise de masse musculaire (e.g., Olivardia, 2001 ; Pope et al., 1997), et cela s'accompagne de comportements dysfonctionnels visant à diminuer cette insatisfaction corporelle (Pope et al., 1997). Cette pathologie a d'abord été catégorisée dans les Troubles du Comportement Alimentaire (TCA) dans le DSM-IV-TR (2000), puis dans les Troubles Obsessionnels Compulsifs (TOC) dans le DSM-V-TR (2015). Certains auteurs continuent à considérer la DM comme un TCA (e.g., Compte, Sepulveda, \& Torrente, 2015 ; Murray et al., 2013 ; Nieuwoudt, Zhou, Coutts, \& Booker, 2015), ces deux pathologies ayant le même cœur 
pathologique (i.e., une intolérance à la détresse, une faible estime de soi et une présence de perfectionnisme), à l'exception des problèmes interpersonnels. Néanmoins, d'autres auteurs considèrent que les perturbations alimentaires sont secondaires dans le diagnostic et que la DM est davantage une préoccupation par rapport à un aspect spécifique du corps (Olivardia, 2001). La DM aurait alors une comorbidité importante avec le trouble de dysmorphie corporelle et les troubles obsessionnels compulsifs (e.g., Chandler, Derryberry, Grieve, \& Pegg, 2009 ; Maida \& Armstrong, 2005 ; Nieuwoudt et al., 2015 ; Olivardia, 2001 ; Olivardia et al., 2000 ; Pope et al., 1997). Pour ces auteurs, les hommes atteints de DM auraient des comportements obsessionnels tels que les rituels de diètes aboutissant à des conduites alimentaires problématiques, et des entraînements excessifs et compulsifs, dans l'optique d'augmenter leur masse musculaire.

La littérature actuelle est abondante en ce qui concerne la recherche de prise de masse musculaire et plus largement la dysmorphie musculaire chez les sportifs masculins, et notamment chez les bodybuilders. Plusieurs revues de questions dont trois méta-analyses, ont été réalisées ces dernières années en relation avec ces thématiques. Une première catégorie identifie la DM comme TCA chez les hommes (e.g., Chapman \& Woodman, 2015 ; Leone et al., 2005 ; Mitchison \& Mond, 2015 ; Murnen \& Karazsia, 2017), ou répertorie les outils de mesure de la recherche de prise de masse musculaire (e.g., Edwards, Tod, \& Molnar, 2014). Une deuxième catégorie se centre sur les critères de diagnostic de la DM (e.g., Behar \& Arancia, 2015 ; dos Santos Filho et al., 2016 ; Lopez-Cuautle et al., 2016). Une troisième catégorie s'intéresse aux conséquences comportementales de la DM en termes de stratégies nutritionnelles (Contesini et al., 2013), de consommation de substances illicites (GarciaRodriguez et al., 2017) et de dépendance à l'exercice (Tod \& Edwards, 2015). Enfin la récente méta-analyse de Mitchell et al. (2017b) s'est intéressée aux symptômes de la DM, et plus précisément aux différences entre les bodybuilders non-compétiteurs et compétiteurs. 
101 Néanmoins, aucune revue de question n’a été réalisée en ce qui concerne les facteurs sociodémographiques, socioculturels, et psychologiques, ou encore les comportements et les troubles, associés à la recherche de prise de masse musculaire et à la DM. L'objectif de cette étude était ainsi de réaliser une revue des travaux sur ces objets afin de compléter la littérature existante.

\section{Méthode}

Sélection des articles. Une méthodologie classique de revue a été utilisée pour cette analyse de la littérature. Le chercheur principal a mené une recherche de la littérature anglophone existante dans GoogleScholar, PubMed, ScienceDirect (à partir de 1995 jusqu'en 2017). L'équation de recherche était la suivante: (« dysmorphia » ou «drive for muscularity » ou «bodybuilding ») et «psychological factors » et «bigorexy » et « disordered eating » et « doping ». La recherche bibliographique a été complétée par une recherche manuelle des références citées dans les articles identifiés. Les critères d'inclusion étaient les suivants : (a) études examinant la prévalence de la recherche de prise de masse musculaire ou de la dysmorphie musculaire ; (b) études examinant les facteurs psychologiques, socioculturels ou sociodémographiques associés à la recherche de prise de masse musculaire, à la dysmorphie musculaire ou à la pratique du bodybuilding ; (c) études évaluant les conséquences de la pratique du bodybuilding ou de la recherche de prise de masse musculaire, ou plus largement, de la dysmorphie musculaire. Les études portant sur les troubles généraux du comportement alimentaire (i.e., anorexie nerveuse, anorexie athlétique, boulimie nerveuse), les recherches portant sur le développement et la validation d'outils de mesure, celles utilisant des outils non valides scientifiquement et les thèses non publiées ont été exclues.

Extraction des données. L'interrogation des différentes bases de données a permis d'identifier un total de 130 études. Quinze études supplémentaires ont été retenues à partir de 
126 la recherche manuelle. Après l'analyse des résumés et l'élimination des doublons, 86 études

127 ont été retenues (identifiables dans les références) : 39 études portent sur les comportements

128 et les troubles associés à la recherche de prise de masse musculaire et à la DM, 10 études sur

129 les facteurs sociodémographiques, 19 études sur les facteurs socioculturels et 18 études sur les

130 facteurs psychologiques associés. Parmi les études quantitatives retenues, des revues de

131 questions $(n=14)$, ainsi que des méta-analyses $(n=4)$ ont été identifiées. Les autres études

132 sont majoritairement observationnelles, englobant des études descriptives $(n=31)$ et des

133 études corrélationnelles $(n=29)$; de rares études expérimentales $(n=5)$ ont également été

134 identifiés. La plupart de ces études sont transversales. Enfin, trois études qualitatives ont été

135 incluses. Les données relatives à chaque article, à savoir, auteur(s), date de publication, type

136 d'étude et nombre de sujets ayant participé à l'étude, ont été reportées (Tableau 1).

137

138 Résultats

139 Les résultats sont présentés selon les quatre catégories d'études identifiées : (a) les

140 comportements et les troubles associés à la recherche de prise de masse musculaire et à la

$141 \mathrm{DM}$; (b) les facteurs sociodémographiques ; (c) les facteurs socioculturels ; et (d) les facteurs

142 psychologiques associés.

143 Comportements et troubles associés à la recherche de prise de masse musculaire et à la

144 DM

Plusieurs types de conséquences comportementales négatives, reliées à la recherche de

146 prise de masse musculaire et à la dysmorphie musculaire, ont été identifiées dans la littérature

147 (e.g., Chittester \& Hausenblas, 2009 ; Hildebrandt, Schlundt, Langenbucher, \& Chung, 2006 ;

148 Leone et al., 2005 ; Lopez, Pollack, Gonzales, Pona, \& Lundgren, 2015 ; Murray et al., 2012 ;

149 Tod \& Edwards, 2015) : (a) des troubles de l'alimentation ; (b) l'utilisation de suppléments 
alimentaires amenant au dopage ; (c) une dépendance à l'exercice ; (d) une diminution des activités sociales.

Troubles du comportement alimentaire. La recherche de prise de masse musculaire a été reliée au développement de comportements alimentaires déviants (e.g., Babusa \& Túry, 2012 ; Murray et al., 2010 ; Pope et al., 1997). La recherche de prise de masse musculaire est associée à la pratique de diètes restrictives où l'ingestion de sucre et l'apport calorique est très réduit (e.g., Helms et al., 2014 ; Gentil, 2015), ce qui augmenterait les risques de développer des troubles du comportement alimentaire (e.g., Mosley, 2009). Plusieurs études (e.g., Mitchell et al., 2017a) mettent en évidence que la recherche de prise de masse musculaire est associée à la mise en place d'un régime stressant, possiblement associé à une haute teneur en protéines et une très basse teneur en graisse (e.g., Consetini et al., 2013 ; Leone et al., 2005), aboutissant parfois à des accès hyperphagiques (e.g., Hallsworth et al., 2005).

Dopage. Parallèlement, plusieurs études ont montré que la recherche de prise de masse musculaire et le développement de la DM aboutissaient à l'utilisation de suppléments alimentaires (e.g., Consetini et al., 2013 ; Froiland et al., 2004 ; Mitchell et al., 2017a) et de substances illicites (e.g., Babusa \& Túry, 2012 ; Bahri et al., 2017 ; Cafri, van den Berg, \& Thompson, 2006 ; García-Rodríguez, Alvarez-Rayón, Camacho-Ruíz, Amaya-Hernández, \& Mancilla-Díaz, 2017 ; Olivardia et al., 2000).

Les produits dopants les plus utilisés sont les stéroïdes anabolisants (79.4\%) (Haerinejad, Ostovar, Farzaneh, \& Keshavarz, 2016), et plus précisément, le Déca-durabolin $(57.6 \%)$ et la testostérone (52\%). Les bodybuilders sont des sportifs particulièrement à risque d'utiliser le dopage (e.g., Bahri et al., 2017) et plus particulièrement les bodybuilders compétiteurs (e.g., Baghurst \& Lirgg, 2009). Une étude réalisée par Parkinson et Evans (2006) a montré que 78.4 \% des utilisateurs de stéroïdes anabolisants s'entraînent de façon récréative et utilisent les produits dopants seulement pour améliorer leur esthétique. De 
nombreuses personnes ingèrent des doses plus élevées que les recommandations, ce qui peut les prédisposer à une variété de problèmes de santé tels qu'une fermeture de l'épiphyse prématurée, une augmentation de la pression du sang, une tumeur du foie, de la stérilité, un élargissement de la prostate, un taux élevé de cholestérol, une insuffisance rénale, etc. (e.g., Pope \& Katz, 1994).

Dépendance sportive. La dépendance à l'exercice se réfère à un souci de pratique accompagné par des sentiments de culpabilité et d'anxiété lorsqu'il est impossible de faire de l'exercice (Hale, Roth, DeLong, \& Briggs, 2010). La dépendance à l'exercice peut coexister avec un trouble de l'alimentation (e.g., Hale et al., 2010). Il a été montré dans de nombreuses études que les bodybuilders sont des sportifs particulièrement dépendants de leur pratique sportive (e.g., Emini \& Bond, 2014), et plus particulièrement les bodybuilders compétiteurs (e.g., Skemp et al., 2013). La recherche de prise de masse musculaire peut mener certains hommes à s'entraîner de manière intensive, durant de longues périodes, sans récupération adéquate, ce qui les expose à des risques de blessures (Olivardia, 2001).

Dans une étude réalisée sur des haltérophiles (Olivardia et al., 2000), 50 \% de ceux ayant de la DM ont déclaré passer plus de trois heures par jour à penser à leur masse musculaire, $58 \%$ ont rapporté avoir une activité «modérée » ou « sévère » et éviter certains lieux, ou personnes, en raison de leurs défauts corporels perçus. Un total de $54 \%$ de ces haltérophiles pensent avoir « peu » ou « aucun » contrôle sur leur compulsion à pratiquer 1'haltérophilie et leurs régimes alimentaires. Sur 24 sujets, deux d'entre eux disent avoir abandonné leurs emplois bien rémunérés pour travailler dans des salles où ils peuvent soulever des poids eux-mêmes (Olivardia et al., 2000).

Isolement social. La DM peut aboutir à un dysfonctionnement social, professionnel ou récréatif, tel que le refus d'invitations sociales pour éviter de devoir exposer son corps, perçu comme inadéquat (Olivardia et al., 2000). Ainsi, les hommes focalisés sur la recherche de 
prise de masse musculaire ont tendance à diminuer leurs activités sociales, afin de consacrer

201 tout leur temps à des exercices routiniers où l'entraînement en musculation est central (e.g.,

202 Maida \& Armstrong, 2005 ; Pope et al., 1997). Certains auteurs, tels que Tod et Edwards

203 (2015) ou Lopez et al. (2015), ont associé la DM à une faible qualité de vie. Des tentatives de

204 suicide ont été observées chez les individus qui sont atteints de DM (Bjorsnsson et al., 2013),

205 et d'autant plus chez ceux qui utilisent des stéroïdes anabolisants (Mosley, 2009).

Au-delà de l'étude des comportements et des troubles associés à la recherche de prise

207 de masse musculaire et à la DM, d'autres travaux se sont intéressés aux facteurs

208 sociodémographiques, socioculturels et psychosociaux.

\section{Facteurs sociodémographiques}

Plusieurs facteurs sociodémographiques ont été mis en relation avec la recherche de

212 prise de masse musculaire et la dysmorphie musculaire, tels que (a) l'âge, (b) le sexe et

213 l'orientation sexuelle, (c) la race et l'origine ethnique, (d) le niveau d'expertise.

Age. Frederick et al. (2007) ont observé que 90 à $95 \%$ des étudiants sont insatisfaits

215 de leur corps et désirent être plus musclés. Plus récemment, Valls, Bonvin et Chabrol (2013)

216 ont trouvé que $85 \%$ des hommes universitaires français étaient insatisfaits de leur masse

217 musculaire. Bien que la DM puisse commencer à un âge précoce, le trouble dysmorphique

218 corporel pourrait apparaitre dans l'enfance ou l'adolescence, et l'âge moyen d'apparition

219 serait entre 16 et 17 ans (Bjornsson et al., 2013). En effet, l'étude de Longobardi, Prino,

220 Fabris, et Settanni (2017) a montré que les jeunes bodybuilders étaient plus à risque de

221 développer de la DM.

Sexe et orientation sexuelle. Des différences liées au sexe ont été rapportées. Les

223 hommes expriment généralement un niveau plus élevé de recherche de prise de masse

224 musculaire, comparativement aux femmes, et les homosexuels seraient particulièrement 
caractérisés par le désir d'augmenter leur masse musculaire (e.g., Hoffmann \& Warschburger,

2262017 ; Olivardia et al., 2000 ; Robert, Munroe-Chandler, \& Gammage, 2009 ; Zelli, Lucidi, \&

227 Mallia, 2010). L'étude d'Olivardia et al. (2000) a montré que $21 \%$ des haltérophiles atteints

228 de DM étaient homosexuels, contre $17 \%$ des haltérophiles n'étant pas atteints.

Race et origine ethnique. Les individus développeraient différents idéaux corporels en

230 fonction de leur culture d'origine (e.g., Cheng, McDermott, Wong, \& La, 2016), ce qui

231 expliquerait notamment pourquoi les hommes américains ont des niveaux de recherche de

232 prise de masse musculaire plus élevés que les hommes chinois (Jung, Forbes, \& Chan, 2010).

233 D'après Baghurst et Kissinger (2009), les hommes de type caucasien désirent atteindre un

234 physique maigre et musclé, alors que les autres populations ont un poids plus élevé et

235 paraissent se satisfaire de ce poids. Ainsi, les caucasiens ont rapporté plus de symptômes

236 reliés à la DM, que les afro-américains (Baghurst \& Kissinger, 2009).

Niveau d'expertise. Plusieurs études ont mis en évidence que la dysmorphie

238 musculaire serait principalement présente chez les hommes sportifs, les athlètes ayant des

239 niveaux de recherche de prise de masse musculaire plus élevés que les non-athlètes (e.g., Zelli

240 et al., 2010). Des recherches antérieures ont suggéré que les athlètes masculins pratiquant des

241 sports avec des poids (e.g., Hernández-Martínez, González-Martí, \& Jordán, 2016 ;

242 Nieuwoudt et al., 2015), tels que le bodybuilding, étaient plus à risque de développer des

243 troubles du comportement alimentaire (e.g., Raevuori et al., 2006). De plus, la méta-analyse

244 de Mitchell et al. (2017b) s'est intéressée à la différence entre les bodybuilders non-

245 compétiteurs et les bodybuilders compétiteurs. Les principaux résultats montrent que les

246 bodybuilders compétiteurs ont des indices de symptomatologie de la DM plus élevés que les

247 bodybuilders non-compétiteurs sur de nombreux points, tels que l'adoption de comportements

248 alimentaires diététiques (ES range 0.66-1.96, $p=0.001$ ) ; l'utilisation de suppléments

249 alimentaires (ES range $0.1-2.35, p=0.001)$; l'utilisation de produits dopants (ES range -0.1 
to $0.99, p=0.001)$; les risques de blessure $(\mathrm{ES}$ range $0.9-1.25, p=0.001)$; la dépendance à

251 l'activité sportive (ES range $0.03-2.15, p=0.006)$; l'utilisation excessive des miroirs (ES range $0.8-1.2, p=0.001)$; et la relation négative à l'indice de masse grasse (ES range -0.87 to $-1.93, p=0.001)$.

\section{Facteurs socioculturels}

Certains facteurs socioculturels et environnementaux ont été reliés à la recherche de prise de masse musculaire et à la dysmorphie musculaire, tels que (a) les normes sociétales et (b) les influences sociales.

Normes sociétales. Les médias, et particulièrement les médias des pays anglo-saxons,

260 véhiculent de plus en plus le message que le corps mésomorphique est un symbole de 261 bonheur, de désirabilité et de succès, et qu'il est impératif pour les hommes de l'atteindre (e.g., Dryer, Farr, Hiramatsu, \& Quinton, 2016 ; McLean, Paxton, \& Wertheim, 2016).

263 Plusieurs études ont rapporté les effets de l'exposition à des publicités sur la satisfaction 264 corporelle et l'estime de soi (e.g., Barlett, Vowels, \& Saucier, 2008 ; Farquhar \& Wasylkiw, 2652007 ; Galioto \& Crowther, 2013 ; Slater \& Tiggemann, 2014). Certains auteurs ont trouvé 266 que l'exposition à des images fixes, ou à des images en mouvement n'avaient pas la même 267 influence sur les hommes (e.g., Hatoum \& Belle, 2004 ; Smolak \& Stein, 2006). En effet, les 268 individus qui regardent les images statiques d'un physique idéalisé peuvent évaluer le 269 physique de l'image et le comparer à leur propre corps, alors que cela se fait moins facilement 270 avec des images en mouvement (e.g., Hatoum \& Belle, 2004 ; Smolak \& Stein, 2006). En plus de faire la promotion d'un idéal masculin musclé, les médias semblent suggérer aux hommes que les femmes apprécient davantage les hommes correspondant à cet

273 idéal, alors que les femmes paraissent moins sévères dans leur jugement (e.g., Grossbard, 274 Neighbors, \& Larimer, 2011). En effet, les femmes seraient moins exigeantes envers l'idéal 
masculin car les modèles masculins présentés dans les magazines féminins seraient moins

276 musclés, que les modèles des magazines masculins (e.g., Grossbard et al., 2011). Cela est

277 conforme aux résultats trouvés dans l'étude de Pope et al. (1999) où les hommes ont choisi un

278 corps idéal d'environ 12.7 kg plus musclé qu'eux-mêmes, et ont estimé que les femmes

279 préféraient un corps masculin d'environ 13.6 kg plus musclé qu'eux-mêmes.

$280 \quad$ Influences sociales. Parallèlement aux normes sociétales, les jeunes hommes

281 subissent des influences sociales de la part de leurs pairs ou de leurs proches, qui peuvent

282 avoir des répercussions sur la perception qu'ils ont d'eux-mêmes (e.g., Lin \& DeCusati,

2832016 ; McCabe et al., 2015). Des études ont montré que la pression perçue pour être plus

284 musclé, les commentaires et les comportements du père, prédisent le désir d'augmenter la

285 masse musculaire et l'adoption de stratégies pour prendre de la masse musculaire chez les

286 jeunes hommes (e.g., Galioto, Karazsia, \& Crowther, 2012 ; Smolak, Murnen, \& Thompson,

287 2005). Par ailleurs, chez des haltérophiles atteints de DM, il a été démontré que $29 \%$ d'entre

288 eux, contre 3\% d'haltérophiles n'ayant pas cette pathologie, ont été spectateurs de violence

289 entre leur père et leur mère «parfois » ou « souvent » pendant l'enfance. La violence entre

290 leur mère et eux-mêmes a aussi été signalée comme survenant «parfois » ou « souvent » pour

$29133 \%$ des sujets avec de la DM, contre $3 \%$ des sujets sans DM (Olivardia et al., 2000). Enfin,

292 les pairs semblent avoir une place importante quant à l'insatisfaction corporelle des jeunes

293 hommes, par un processus de modélisation ou par un processus de conformité au groupe

294 d'appartenance (e.g., Galioto et al., 2012 ; Smolak et al., 2005).

\section{Facteurs psychologiques}

Plusieurs facteurs psychologiques personnels ont été associés à la recherche de prise

298 de masse musculaire et à la dysmorphie musculaire, tels que (a) l'insatisfaction corporelle et

299 (b) les traits de personnalité. 
Insatisfaction corporelle. Les hommes souffrant d'insatisfaction corporelle ont

tendance à se percevoir comme moins attirants physiquement, moins forts et moins

performants dans les sports que les autres hommes (e.g., Gonzáles-Martí, Bustos, Hernándes-

Martínez, \& Jordàn, 2014). Olivardia et al. (2004) ont fait la distinction entre l'insatisfaction

corporelle par rapport aux muscles et par rapport à la masse grasse, il en a résulté que chez les

hommes, l'évaluation de soi et le concept de soi reposeraient davantage sur l'évaluation de la masse musculaire, plutôt que sur l'impression d'être trop gras. Plusieurs études ont montré le lien entre l'insatisfaction corporelle et le développement des TCA (e.g., Dakanalis et al., 2015b), et plus particulièrement entre l'insatisfaction corporelle et le développement de la DM (e.g., Mącik \& Kowalska-Dąbrowska, 2015). L'étude menée par Olivardia et al. (2000) a relaté que $52 \%$ des haltérophiles atteints de DM ont répondu être « en désaccord » ou « un

311 peu en désaccord » avec l'item : «J'aime vraiment mon corps », contre $20 \%$ des haltérophiles non atteints de la pathologie.

Des études réalisées sur des bodybuilders en salle de musculation ont montré que les sportifs très insatisfaits corporellement ont tendance à utiliser les miroirs pour vérifier leur 315 apparence (e.g., Mosley, 2009). De plus, une variabilité dans les symptômes de la DM selon 316 l'horaire d'entraînement des hommes a été trouvée dans l'étude de Thomas, Tod et Lavallé 317 (2011) : les sportifs apparaissent plus insatisfaits corporellement lors d'un jour de repos, 318 plutôt qu'un jour d'entraînement physique.

Traits de personnalité. Plusieurs traits de personnalité tels que l'anxiété, la dépression

320 et le névrosisme ont été positivement associés à la recherche de prise de masse musculaire 321 (e.g., Boyda \& Shevlin, 2011 ; Davis, Karvinen, \& McCreary, 2005 ; Kuennen \& Waldrom, 2007 ; Maida \& Armstrong, 2005 ; Wolke \& Sapouna, 2008), alors que l'estime de soi apparait reliée négativement (e.g., Gonzalez-Marti et al., 2014 ; Kuennen \& Waldrom, 2007 ; 
avec DM avaient de l'anxiété, un passé dépressif et étaient bipolaires, contre $20 \%$ des

326 haltérophiles sans DM. De plus, $29 \%$ ont déclaré avoir une histoire de vie incluse dans l'axe

327 des troubles de l'anxiété du DSM-IV, contre $3 \%$ des haltérophiles n'étant pas atteints de DM.

328 Ces résultats sont consistants avec ceux récemment rapportés par Longobardi et al. (2017),

329 dans lesquels les bodybuilders à risque de DM rapportaient des symptômes de dépression et 330 d'anxiété plus élevés.

Le névrosisme aurait un rôle d'amplificateur émotionnel qui augmenterait les affects

332 négatifs et rendrait plus à risque le développement de comportements extrêmes dont la DM

333 (Davis et al., 2005). Il serait associé positivement à l'insatisfaction corporelle (Allen \&

334 Walter, 2016) et à la recherche de prise de masse musculaire (e.g., Benford \& Swami, 2014).

L'extraversion serait associée négativement à l'insatisfaction corporelle (e.g., Allen \&

336 Walter, 2016 ; Benford \& Swami, 2014), alors que l'ouverture et l'agréabilité ne seraient pas

337 liées à l'image corporelle (Allen \& Walter, 2016).

Les études ayant examiné les relations entre le perfectionnisme et la DM sont

339 globalement consistantes et montrent des relations directes positives (e.g., Dakanalis et al.,

340 2015a ; Kuennen \& Waldrom, 2007 ; Murray et al., 2013). Enfin certaines recherches

341 s'intéressant au narcissisme, ont révélé une absence de relation avec la DM (e.g., Collis,

342 Lewis, \& Crisp, 2016 ; Davis et al., 2005 ; Kuennen \& Waldrom, 2007).

\section{Discussion générale}

L'objectif de ce travail était de recenser l'ensemble des articles scientifiques publiés

346 dans des revues internationales anglophones, et portant sur la recherche de prise de masse

347 musculaire et la dysmorphie musculaire. Notre requête et notre analyse ont permis d'identifier

34886 articles répondant aux critères d'inclusion. Ces études sont principalement anglo-saxonnes,

349 de nature observationnelle (i.e., études descriptives et corrélationnelles) et transversales. Les 
études les moins nombreuses concernent les facteurs sociodémographiques associés à la recherche de prise de masse musculaire et à la DM $(n=10)$. Un nombre quasiment équivalent

d'études concerne les facteurs socioculturels $(n=19)$ et psychologiques $(n=18)$ de la

353 recherche de prise de masse musculaire et de la DM. Les recherches portant sur les

354 comportements et les troubles reliés à la recherche de prise de masse musculaire et à la DM

355 sont les plus nombreuses $(n=39)$. Après avoir discuté l'apport de ces études, nous

356 identifierons un ensemble de limites appelant à des pistes de recherche futures.

En premier lieu, les études portant sur les facteurs sociodémographiques associés à la

DM montrent que les populations d'origine caucasienne seraient les plus vulnérables à la recherche de prise de masse musculaire et à la DM, ainsi que les jeunes sportifs masculins

361 pratiquant des sports de poids (e.g., Bjornsson et al., 2013 ; Cheng et al., 2015 ; Hoffmann \& Warschburger, 2017 ; Longobardi et al., 2017). Ces résultats sont en accord avec la métaanalyse de Chapman et Woodman (2015), qui a montré que les athlètes pratiquant des sports

364 qui mettent l'accent sur la forme du corps et sur une faible masse corporelle, avaient des 365 niveaux de TCA plus élevés que les hommes qui ne pratiquaient pas d'activité sportive. Ils 366 corroborent également la récente méta-analyse de Mitchell et al. (2017b) qui a mis en 367 évidence que le niveau d'expertise était un facteur de risque, les bodybuilders compétiteurs 368 étant plus à risque de développer des symptômes de la DM, que les bodybuilders non369 compétiteurs. De futures études pourraient investiguer la prévalence de la recherche de prise 370 de masse musculaire et de DM selon les catégories socioprofessionnelles et le statut marital. 371 En deuxième lieu, et concernant les facteurs socioculturels, notre analyse a permis de 372 montrer que la pression médiatique était un facteur de risque récurrent de recherche de prise 373 de masse musculaire et de développement de DM (e.g., Cramblitt \& Pritchard, 2013 ; Galioto 374 \& Crowther, 2013 ; Pritchard \& Cramblitt, 2014 ; Slater \& Tiggemann, 2014). Ces données 
convergent vers les conclusions de la revue systématique de McLean, Paxton et Werteim

376 (2016) portant sur l'influence des médias sur les TCA. De plus, de nombreuses études observationnelles ont mis en évidence que les influences sociales, notamment en provenance des pairs, étaient des prédicteurs significatifs des symptômes de la DM (e.g., Dryer, Farr, Hiramatsu, \& Quinton, 2016 ; Lin \& DeCusati, 2016 ; Schneider, Rollitz, Voracek, \& Hennig-Fast, 2016). Des travaux ultérieurs pourront examiner l'influence relative des médias,

381 de la famille et des pairs sur la DM, via l'insatisfaction corporelle, ce qui pourrait permettre la 382 généralisation du modèle de Schroff et Thompson (2006) portant sur les TCA, à la DM. En troisième lieu, en ce qui concerne les facteurs psychologiques, notre analyse a

384 permis d'identifier que l'insatisfaction corporelle était un des principaux facteurs

385 psychologiques associés à la recherche de prise de masse musculaire (Murnen \& Karazsia, 386 2017) et à la DM (Dakanalis et al., 2015b). Par ailleurs, certains traits de personnalité tels que 387 l'anxiété, la dépression, le perfectionnisme et le névrosisme seraient des facteurs de risque de 388 la recherche de prise de masse musculaire ou de DM, alors que l'estime de soi élevée serait un 389 facteur protecteur (e.g., Allen \& Walter, 2016 ; Benford \& Swami, 2014 ; Dakanalis et al., 390 2015a ; Longobardi et al., 2017). Ces différentes études sont majoritairement exploratoires et 391 ne semblent pas avoir été fondées de manière théorique (Edwards et al., 2014). Par ailleurs, aucune d'entre elles n'a exploré les facteurs motivationnels, ni les croyances comportementales. De plus, les études portant sur les facteurs psychosociaux reliés à la

394 recherche de prise de masse musculaire et à la DM se sont seulement intéressées à des 395 processus explicites. Ainsi, de futurs travaux pourraient s'appuyer sur les modèles intégratifs 396 contemporains de la motivation ou de la santé (e.g., Hagger \& Chatzisarantis, 2009, 2014 ; 397 Hoffmann, Friese, \& Wiers, 2008) pour appréhender les mécanismes psychosociaux 398 explicites et implicites sous-tendant la DM. Enfin, les tailles d'effet concernant les facteurs 399 psychologiques ayant été très peu explorées, contrairement aux comportements et troubles 
associés à la recherche de prise de masse musculaire et à la DM (Mitchell et al., 2017b), une

401 méta-analyse pourrait être réalisée à cette fin.

Comme précisé précédemment, les études les plus nombreuses concernent les

comportements déviants associés à la recherche de prise de masse musculaire et à la DM.

404 Différents types de conséquences négatives ont été répertoriées concernant les TCA,

405 l'utilisation de suppléments alimentaires et le dopage, la dépendance à l'exercice et la

406 diminution des activités sociales. Ces différentes catégories d'études sont conformes aux

407 méta-analyses antérieures respectives de Contesini et al. (2013), Garcia-Rodriguez et al.

408 (2017) et Tod et Edwards (2015). L'originalité de notre revue de littérature est de montrer que

409 la DM est susceptible d'avoir des conséquences délétères sur les relations sociales avec un

410 risque d'isolement (Olivardia et al., 2000) et de troubles psychologiques graves pouvant aller

411 jusqu'à des tentatives de suicide (Bjornsson et al., 2013). Des études complémentaires sont

412 toutefois nécessaires pour mieux saisir la dynamique des relations sociales et du soutien social

413 des bodybuilders et prévenir leur potentiel isolement.

$414 \quad$ Plus globalement, les études existantes étant principalement transversales et

415 corrélationnelles, de nouvelles recherches de nature expérimentale et longitudinale sont

416 requises pour établir des relations de cause à effet entre les facteurs identifiés et la DM. Par

417 exemple, la question se pose de savoir si le bodybuilding est une cause de la DM ou si le

418 bodybuilding attire les individus qui sont prédisposés à la DM. Une étude longitudinale

419 pourrait permettre d'identifier comment les symptômes de la DM apparaissent au cours de

420 l'engagement dans la pratique. Enfin, les outils permettant de mesurer la recherche de prise de

421 masse musculaire semblent avoir été fondés de manière a-théorique pour la plupart (e.g.,

422 McCreary \& Sasse, 2000), et aucune version française n'existe jusqu'alors ; c'est pourquoi, il

423 serait nécessaire d'envisager le développement et la validation d'un nouvel outil en langue

424 française fondé théoriquement. Plus largement, il serait important de conduire de nouvelles 
425 études auprès de populations autres qu'anglophones, afin de comparer les résultats aux

426 recherches anglophones.

Au-delà de l'état des connaissances scientifiques sur le sujet, cette revue de question

sur les facteurs sociodémographiques, socioculturels, et psychologiques associés à la

429 recherche de prise de masse musculaire et de DM, présente l'intérêt de suggérer un ensemble

430 de pistes de prévention auprès des sportifs s'engageant dans la pratique intensive du

431 bodybuilding. Outre la détection de personnalité et d'environnement social à risque, il semble

432 important, à terme, de mener une réflexion sur les normes corporelles masculines valorisées

433 dans notre société, afin de promouvoir chez les jeunes générations un engagement dans

434 l'activité physique et/ou sportive motivé par des fins de santé et d'accomplissement

435 personnel, plus que d'esthétisme ou de « performance à tout prix ».

436

\section{Bibliographie}

438 Allen, M. S., \& Walter, E. E. (2016). Personality and body image: A systematic review. Body image, 19, 79-88.

Amadieu, J. F. (2002). Poids des apparences (Le): Beauté, amour et gloire. Paris : Odile Jacob.

American Psychiatric Association, Diagnostic and Statistical Manual of Mental Disorders, Association; 2000.

American Psychiatric Association. (2013). Diagnostic and statistical manual of mental disorders (DSM-5®). American Psychiatric Pub.

Babusa, B., \& Túry, F. (2012). Muscle dysmorphia in Hungarian non-competitive male bodybuilders. Eating and Weight Disorders-Studies on Anorexia, Bulimia and Obesity, 17(1), e49-e53. 
Baghurst, T., \& Kissinger, D. B. (2009). Current and future perspectives of muscle dysmorphia. International Journal of Men's Health, 8, 82-89.

452 453

Baghurst, T., \& Lirgg, C. (2009). Characteristics of muscle dysmorphia in male football, weight training, and competitive natural and non-natural bodybuilding samples. Body Image, 6(3), 221-227.

Bahri, A., Mahfouz, M. S., Marran, N. M., Dighriri, Y. H., Alessa, H. S., Khwaji, M. O., \& Zafar, S. M. (2017). Prevalence and awareness of anabolic androgenic steroid use among male body builders in Jazan, Saudi Arabia. Tropical Journal of Pharmaceutical Research, 16(6), 1425-1430.

Barlett, C. P., Vowels, C. L., \& Saucier, D. A. (2008). Meta-analyses of the effects of media images on men's body-image concerns. Journal of Social and Clinical Psychology, 27(3), 279-310.

Behar, R., \& Arancibia, M. (2015). Body image disorders: anorexia nervosa versus reverse anorexia (muscle dysmorphia). Revista Mexicana de Trastornos Alimentarios, 6(2), 121-128.

Benford, K., \& Swami, V. (2014). Body image and personality among British men: Associations between the Big Five personality domains, drive for muscularity, and body appreciation. Body image, 11(4), 454-457.

Bjornsson, A. S., Didie, E. R., Grant, J. E., Menard, W., Stalker, E., \& Phillips, K. A. (2013). Age at onset and clinical correlates in body dysmorphic disorder. Comprehensive Psychiatry, 54(7), 893-903.

Boyda, D., \& Shevlin, M. (2011). Childhood victimisation as a predictor of muscle dysmorphia in adult male bodybuilders. The Irish Journal of Psychology, 32(3-4), 105-115. 
474 Cafri, G., van den Berg, P., \& Thompson, J. K. (2006). Pursuit of Muscularity in Adolescent

475

476

477

478

479

480

481

482

483

484

485

486

487

488

489

490

491

492

493

494

495

496 Boys : Relations Among Biopsychosocial Variables and Clinical Outcomes. Journal of Clinical Child \& Adolescent Psychology, 35(2), 283-291.

Cella, S., Iannaccone, M., \& Cotrufo, P. (2012). Muscle dysmorphia: A comparison between competitive bodybuilders and fitness practitioners. Journal of Nutritional Therapeutics, 1(1), 12-18.

Chandler, C. G., Derryberry, W. P., Grieve, F. G., \& Pegg, P. O. (2009). Are Anxiety and Obsessive-Compulsive Symptoms Related to Muscle Dysmorphia? International Journal of Men's Health, 8(2), 143-154.

Chapman, J., \& Woodman, T. (2015). Disordered eating in male athletes: a meta-analysis. Journal of Sports Sciences, 1-9.

Cheng, H. L., McDermott, R. C., Wong, Y. J., \& La, S. (2016). Drive for muscularity in Asian American men: Sociocultural and racial/ethnic factors as correlates. Psychology of Men \& Masculinity, 17(3), 215.

Chittester, N. I., \& Hausenblas, H. A. (2009). Correlates of drive for muscularity: The role of anthropometric measures and psychological factors. Journal of Health Psychology, 14, $872-877$.

Collis, N., Lewis, V., \& Crisp, D. (2016). When Is Buff Enough? The Effect of Body Attitudes and Narcissistic Traits on Muscle Dysmorphia. The Journal of Men's Studies, 24(2), 213-225.

Compte, E. J., Sepulveda, A. R., \& Torrente, F. (2015). A two-stage epidemiological study of eating disorders and muscle dysmorphia in male university students in Buenos Aires. International Journal of Eating Disorders, 48(8), 1092-1101. 
Contesini, N., Adami, F., Blake, M. D. T., Monteiro, C. B., Abreu, L. C., Valenti, V. E., ... \& de Vasconcelos, F. D. A. G. (2013). Nutritional strategies of physically active subjects with muscle dysmorphia. International archives of medicine, 6(1), 25.

Cramblitt, B., \& Pritchard, M. (2013). Media's influence on the drive for muscularity in undergraduates. Eating behaviors, 14(4), 441-446.

Dakanalis, A., Favagrossa, L., Clerici, M., Prunas, A., Colmegna, F., Zanetti, M. A., \& Riva, G. (2015a). Body dissatisfaction and eating disorder symptomatology: a latent structural equation modeling analysis of moderating variables in 18-to-28-year-old males. The Journal of psychology, 149(1), 85-112.

Dakanalis, A., Zanetti, A. M., Riva, G., Colmegna, F., Volpato, C., Madeddu, F., \& Clerici, M. (2015b). Male body dissatisfaction and eating disorder symptomatology: Moderating variables among men. Journal of Health Psychology, 20(1), 80-90.

Davies, R., Smith, D., \& Collier, K. (2011). Muscle dysmorphia among current and former steroid users. Journal of Clinical Sport Psychology, 5, 77-94.

Davis, C., Karvinen, K., \& McCreary, D. R. (2005). Personality correlates of a drive for muscularity in young men. Personality and Individual Differences, 39(2), 349-359.

dos Santos Filho, C. A., Tirico, P. P., Stefano, S. C., Touyz, S. W., \& Claudino, A. M. (2016). Systematic review of the diagnostic category muscle dysmorphia. Australian \& New Zealand Journal of Psychiatry, 50(4), 322-333.

Dryer, R., Farr, M., Hiramatsu, I., \& Quinton, S. (2016). The Role of Sociocultural Influences on Symptoms of Muscle Dysmorphia and Eating Disorders in Men, and the Mediating Effects of Perfectionism. Behavioral Medicine, 42(3), 174-182.

Edwards, C., Tod, D., \& Molnar, G. (2014). A systematic review of the drive for muscularity research area. International Review of Sport and Exercise Psychology, 7(1), 18-41. 
521 Emini, N. N., \& Bond, M. J. (2014). Motivational and psychological correlates of bodybuilding dependence. Journal of behavioral addictions, 3(3), 182-188.

523 Farquhar, J. C., \& Wasylkiw, L. (2007). Media images of men: Trends and consequences of body conceptualization. Psychology of Men \& Masculinity, 8(3), 145.

Frederick, D. a., Buchanan, G. M., Sadehgi-Azar, L., Peplau, L. A., Haselton, M. G., Berezovskaya, A., \& Lipinski, R. E. (2007). Desiring the muscular ideal: Men's body satisfaction in the United States, Ukraine, and Ghana. Psychology of Men \& Masculinity, 8(2), 103-117.

Frederick, D. a, Fessler, D. M. T., \& Haselton, M. G. (2005). Do representations of male muscularity differ in men's and women's magazines? Body Image, 2(1), 81-86.

531 Froiland, K., Koszewski, W., Hingst, J., \& Kopecky, L. (2004). Nutritional supplement use among college athletes and their sources of information. International journal of sport

534 Galioto, R., \& Crowther, J. H. (2013). The effects of exposure to slender and muscular images on male body dissatisfaction. Body image, 10(4), 566-573.

Galioto, R., Karazsia, B. T., \& Crowther, J. H. (2012). Familial and peer modeling and verbal commentary: Associations with muscularity-oriented body dissatisfaction and body change behaviors. Body Image, 9(2), 293-297.

García-Rodríguez, J., Alvarez-Rayón, G., Camacho-Ruíz, J., Amaya-Hernández, A., \& Mancilla-Díaz, J. M. (2017). Muscle dysmorphia and use of ergogenics substances. A systematic review. Revista Colombiana de Psiquiatría (English ed.), 46(3), 168-177.

542 Gentil, P. (2015). A nutrition and conditioning intervention for natural bodybuilding contest preparation: observations and suggestions. Journal of the International Society of Sports Nutrition, 12(1), 1-3. 
545 González-Martí, I., Bustos, J. G. F., Hernández-Martínez, A., \& Jordán, O. R. C. (2014). Physical perceptions and self-concept in athletes with muscle dysmorphia symptoms. The Spanish journal of psychology, 17.

Grossbard, J. R., Neighbors, C., \& Larimer, M. E. (2011). Perceived norms for thinness and muscularity among college students: What do men and women really want?. Eating behaviors, 12(3), 192-199.

Haerinejad, M. J., Ostovar, A., Farzaneh, M. R., \& Keshavarz, M. (2016). The prevalence and characteristics of performance-enhancing drug use among bodybuilding athletes in the south of Iran, Bushehr. Asian journal of sports medicine, 7(3).

Hagger, M. S., \& Chatzisarantis, N. L. (2009). Integrating the theory of planned behaviour and self-determination theory in health behaviour: a meta-analysis. British journal of health psychology, 14(2), 275-302.

Hagger, M. S., \& Chatzisarantis, N. L. (2014). An integrated behavior change model for physical activity. Exercise and Sport Sciences Reviews, 42(2), 62-69.

Hale, B. D., Roth, A. D., DeLong, R. E., \& Briggs, M. S. (2010). Exercise dependence and the drive for muscularity in male bodybuilders, power lifters, and fitness lifters. Body Image, 7, 234-239.

Hallsworth, L., Wade, T., \& Tiggemann, M. (2005). Individual differences in male bodyimage: An examination of self-objectification in recreational body builders. British Journal of Health Psychology, 10, 453-465.

Hatoum, I. J., \& Belle, D. (2004). Mags and abs: Media consumption and bodily concerns in men. Sex Roles, 51(7-8), 397-407.

Helms, E. R., Aragon, A. A., \& Fitschen, P. J. (2014). Evidence-based recommendations for natural bodybuilding contest preparation: nutrition and supplementation. Journal of the International Society of Sports Nutrition, 11(1), 20. 
Hernández-Martínez, A., González-Martí, I., \& Jordán, O. R. C. (2016). Detection of Muscle Dysmorphia symptoms in male weightlifters. Anales de Psicología/Annals of Psychology, 33(1), 204-210.

Hildebrandt, T., Schlundt, D., Langenbucher, J., \& Chung, T. (2006). Presence of muscle dysmorphia symptomology among male weightlifters. Comprehensive Psychiatry, $47(2), 127-135$.

Hoffmann, S., \& Warschburger, P. (2017). Weight, shape, and muscularity concerns in male and female adolescents: Predictors of change and influences on eating concern. International Journal of Eating Disorders, 50(2), 139-147.

Hofmann, W., Friese, M., \& Wiers, R. W. (2008). Impulsive versus reflective influences on health behavior: A theoretical framework and empirical review. Health Psychology Review, 2(2), 111-137.

Jung, J., Forbes, G. B., \& Chan, P. (2010). Global body and muscle satisfaction among college men in the United States and Hong Kong-China. Sex Roles, 63, 104-117.

Kuennen, M. R., \& Waldron, J. J. (2007). Relationships Between Specific Personality Traits, Fat Free Mass Indices, and the Muscle Dysmorphia Inventory. Journal of Sport Behavior, 30(4), 453-470.

Labre, M. P. (2005). The male body ideal: Perspectives of readers and non-readers of fitness magazines. Journal of Men's Health and Gender, 2(2), 223-229.

Lantz, C. D., Rhea, D. J., \& Cornelius, A. E. (2002). Muscle dysmorphia in elite-level power lifters and bodybuilders: a test of differences within a conceptual model. The Journal of Strength \& Conditioning Research, 16(4), 649-655.

Ledoux, M., \& Rivard, M. (2000). Poids corporel Institut de la statistique du Québec, Enquête sociale et de santé (pp. 642). Québec.

Leone, J. E., Sedory, E. J., \& Gray, K. A. (2005). Recognition and treatment of muscle 
dysmorphia and related body image disorders. Journal of Athletic Training, 40(4), $352-9$.

597

598

599

600

601

602

603

604

605

606

607

608

609

610

611

612

613

614

615

616

617

618

619

Lin, L., \& DeCusati, F. (2016). Muscle dysmorphia and the perception of men's peer muscularity preferences. American Journal of Men's Health, 10(6), NP78-NP88.

Longobardi, C., Prino, L. E., Fabris, M. A., \& Settanni, M. (2017). Muscle dysmorphia and psychopathology: Findings from an Italian sample of male bodybuilders. Psychiatry Research, 256, 231-236.

Lopez, A., Pollack, L., Gonzales, S., Pona, A., \& Lundgren, J. (2015). Psychosocial Correlates of Muscle Dysmorphia among Collegiate Males. Journal of Psychological Inquiry, 20(1), 58-66.

Lopez-Cuautle, C., Vazquez-Arevalo, R., \& Mancilla-Díaz, J. M. (2016). Muscle Dysmorphia Diagnostic evaluation: a systematic review. Anales de Psicología/Annals of Psychology, 32(2), 405-416.

Mącik, D., \& Kowalska-Dąbrowska, M. (2015). The risk of muscle dysmorphia and the perception of change in retrospective, current and ideal self-image-preliminary study. Health Psychology Report, 3(1), 24-34.

Maida, D., \& Armstrong, S. (2005). The classification of muscle dysmorphia. International Journal of Men's Health, 4(1), 73-91.

Martínez, S. A., Cortés, C. E., Rizo, B. M., \& Gil, G. V. (2014). Evaluation of gym users'diet with muscle dysmorphia (bigorexia). Nutricion hospitalaria, 32(1), 324-329.

McCabe, M. P., Busija, L., Fuller-Tyszkiewicz, M., Ricciardelli, L., Mellor, D., \& Mussap, A. (2015). Sociocultural influences on strategies to lose weight, gain weight, and increase muscles among ten cultural groups. Body Image, 12, 108-114.

McCreary, D. R., \& Sasse, D. K. (2000). An exploration of the drive for muscularity in adolescent boys and girls. Journal of American college health, 48(6), 297-304. 
McLean, S. A., Paxton, S. J., \& Wertheim, E. H. (2016). The role of media literacy in body dissatisfaction and disordered eating: A systematic review. Body image, 19, 9-23.

Mitchell, L., Hackett, D., Gifford, J., Estermann, F., \& O’Connor, H. (2017a). Do Bodybuilders Use Evidence-Based Nutrition Strategies to Manipulate Physique?. Sports, 5(4), 76.

Mitchell, L., Murray, S. B., Cobley, S., Hackett, D., Gifford, J., Capling, L., \& O’Connor, H. (2017b). Muscle dysmorphia symptomatology and associated psychological features in bodybuilders and non-bodybuilder resistance trainers: A systematic review and meta-analysis. Sports Medicine, 47(2), 233-259.

Mitchison, D., \& Mond, J. (2015). Epidemiology of eating disorders, eating disordered behaviour, and body image disturbance in males: a narrative review. Journal of eating disorders, 3(1), 20.

Mosley, P. E. (2009). Bigorexia: bodybuilding and muscle dysmorphia. European EatingDisorders Review, 17(3), 191-198.

Murnen, S. K., \& Karazsia, B. T. (2017). A review of research on men's body image and drive for muscularity.

Murray, S. B., Rieger, E., Hildebrandt, T., Karlov, L., Russell, J., Boon, E., ... Touyz, S. W. (2012). A comparison of eating, exercise, shape, and weight related symptomatology in males with muscle dysmorphia and anorexia nervosa. Body Image, 9(2), 193-200.

639 Murray, S. B., Rieger, E., Karlov, L., \& Touyz, S. W. (2013). An investigation of the transdiagnostic model of eating disorders in the context of muscle dysmorphia. European Eating Disorders Review, 21(2), 160-164.

Murray, S. B., Rieger, E., Touyz, S. W., \& De la Garza García, Y. (2010). Muscle dysmorphia and the DSM-V conundrum: where does it belong? A review paper. The International Journal of Eating Disorders, 43(6), 483-491. 
645 Nieuwoudt, J. E., Zhou, S., Coutts, R. A., \& Booker, R. (2015). Symptoms of muscle

646

647

648

649

650

651

652

653

654

655

656

657

658

659

660

661

662

663

664

665

666

667

668

669

dysmorphia, body dysmorphic disorder, and eating disorders in a nonclinical population of adult male weightlifters in Australia. The Journal of Strength \& Conditioning Research, 29(5), 1406-1414.

Olivardia, R. (2001). Mirror, Mirror on the Wall, Who's the Largest of Them All ? The Features and Phenomenology of Muscle Dysmorphia. Harvard Review of Psychiatry, 9(5), 254-259.

Olivardia, R., Pope, H. G., \& Hudson, J. I. (2000). Muscle dysmorphia in male weightlifters: A case-control study. The American Journal of Psychiatry, 157(8), 1291-6.

Olivardia, R., Pope Jr., H. G., Borowiecki, J. J., \& Cohane, G. H. (2004). Biceps and Body Image: The Relationship Between Muscularity and Self-Esteem, Depression, and Eating Disorder Symptoms. Psychology of Men \& Masculinity, 5(2), 112-120.

Parkinson, A. B., \& Evans, N. a. (2006). Anabolic androgenic steroids: a survey of 500 users. Medicine and Science in Sports and Exercise, 38(4), 644-651.

Pope, H. G., Gruber, A. J., Choi, P., Olivardia, R., \& Phillips, K. A. (1997). Muscle dysmorphia: An underrecognized form of body dysmorphic disorder. Psychosomatics, $38(6), 548-557$.

Pope, H. G., \& Katz, D. L. (1994). Psychiatric and medical effects of anabolic-androgenic steroid use: a controlled study of 160 athletes. Archives of general psychiatry, 51(5), $375-382$.

Pope, H. G., Katz, D. L., \& Hudson, J. I. (1993). Anorexia nervosa and "reverse anorexia" among 108 male bodybuilders. Comprehensive Psychiatry, 34(6), 406-409.

Pope, H. G., Olivardia, R., Gruber, A., \& Borowiecki, J. (1999). Evolving ideals of male body image as seen through action toys. The International Journal of Eating Disorders, 
671 Pritchard, M., \& Cramblitt, B. (2014). Media influence on drive for thinness and drive for muscularity. Sex Roles, 71(5-8), 208-218.

673 Raevuori, A., Keski-Rahkonen, A., Bulik, C. M., Rose, R. J., Rissanen, A., \& Kaprio, J. (2006). Muscle dissatisfaction in young adult men. Clinical Practice and Epidemiology in Mental Health, 2(1), 6.

676

677

678

679

680

681

682

683

684

685

686

687

688

689

690

691

692

693

Ricciardelli, L. a, \& McCabe, M. P. (2004). A Biopsychosocial Model of Disordered Eating and the Pursuit of Muscularity in Adolescent Boys. Psychological Bulletin, 130(2), 179-205.

Robert, C. A., Munroe-Chandler, K. J., \& Gammage, K. L. (2009). The relationship between the drive for muscularity and muscle dysmorphia in male and female weight trainers. Journal of Strength and Conditioning Research, 23, 1656-1662.

Schneider, C., Rollitz, L., Voracek, M., \& Hennig-Fast, K. (2016). Biological, psychological, and sociocultural factors contributing to the drive for muscularity in weight-training men. Frontiers in psychology, 7.

Shroff, H., \& Thompson, J. K. (2006). The tripartite influence model of body image and eating disturbance: A replication with adolescent girls. Body Image: An International Journal of Research, 3, 17-23.

Skemp, K. M., Mikat, R. P., Schenck, K. P., \& Kramer, N. A. (2013). Muscle dysmorphia: Risk may be influenced by goals of the weightlifter. The Journal of Strength \& Conditioning Research, 27(9), 2427-2432.

Slater, A., \& Tiggemann, M. (2014). Media matters for boys too! The role of specific magazine types and television programs in the drive for thinness and muscularity in adolescent boys. Eating behaviors, 15(4), 679-682. 
694 Smolak, L., Murnen, S. K., \& Thompson, J. K. (2005). Sociocultural influences and muscle building in adolescent boys. Psychology of Men \& Masculinity, 6(4), 227.

Smolak, L., \& Stein, J. A. (2006). The relationship of drive for muscularity to sociocultural factors, self-esteem, physical attributes gender role, and social comparison in middle school boys. Body image, 3(2), 121-129.

Thomas, L. S., Tod, D. A., \& Lavallee, D. E. (2011). Variability in muscle dysmorphia symptoms: The influence of weight training. The Journal of Strength \& Conditioning Research, 25(3), 846-851.

Tod, D., \& Edwards, C. (2015). A meta-analysis of the drive for muscularity's relationships with exercise behaviour, disordered eating, supplement consumption, and exercise dependence. International Review of Sport and Exercise Psychology, 8(1), 185-203.

705

706

Valls, M., Bonvin, P., \& Chabrol, H. (2013). Association between muscularity dissatisfaction and body dissatisfaction among normal-weight French men. Journal of Men's Health, 10(4), 139-145.

Vartanian, L. R., Giant, C. L., \& Passino, R. M. (2001). “Ally McBeal vs. Arnold Schwarzenegger": Comparing mass media, interpersonal feedback and gender as predictors of satisfaction with body thinness and muscularity. Social Behavior and Personality: an international journal, 29(7), 711-723.

Wolke, D., \& Sapouna, M. (2008). Big men feeling small: Childhood bullying experience, muscle dysmorphia and other mental health problems in bodybuilders. Psychology of Sport and Exercise, 9(5), 595-604.

Zelli, A., Lucidi, F., \& Mallia, L. (2010). The relationships among adolescents' drive for muscularity, drive for thinness, doping attitudes, and doping intensions. Journal of Clinical Sport Psychology, 4, 39-52. 